\title{
Incidence Des Fortes Fréquences De Stimulation Hormonale Sur Deux Régimes de Saignée Descendante Du Clone PB 215 : Faible (d6) et Modéré (d4), Au Sud-Est De La Côte D'Ivoire
}

\author{
Coulibaly Lacina Fanlégué \\ Traoré Mohamed Sahabane \\ Université Peleforo GON COULIBALY, UFR Sciences Biologiques, \\ Département de Biologie Végétale, Korhogo, Côte d'Ivoire \\ Kouamé N'dri Marie-Thérèse \\ Université Nangui Abrogoua, Centre de Recherche en Ecologie \\ Abidjan, Côte d'Ivoire
}

Doi: 10.19044/esj.2018.v14n15p499 URL:http://dx.doi.org/10.19044/esj.2018.v14n15p499

\begin{abstract}
The clone PB 235 of Hevea brasiliensis is characterized by a rapid growth, homogeneity, and a virgin bark which is suitable for tapping. However, it is sensitive to the very intense latex systems harvesting technologies. This paper focuses on determining the incidence of strong frequencies of stimulation on two downward tapping systems of clone PB 215: low (d6) or moderate (d4). Rubber trees were planted with the density of 510 trees/ha $(7 \mathrm{~m} \mathrm{x} 2.8 \mathrm{~m})$ in accordance with a randomized complete block design. Tapping at d6 frequency with 8 and 12 hormonal stimulations and the $\mathrm{d} 4$ stimulated 0 and 8 times per year were evaluated. The intensification of hormonal stimulation with the $\mathrm{d} 6 \mathrm{did}$ not have any beneficial effect on the dry rubber yield of the rubber trees. In $\mathrm{d} 4$, the stimulation involved a reduction in the sucrose content which led to a significant increase of the dry rubber yield. However, it contributed to an increase in the tapping panel dryness. At moderate tapping, the application of hormonal stimulation allowed us to distinguish between the two latex harvesting technologies. At d6, the two treatments were similar regardless of the stimulation level. Tapping at d6 supports a reduction of tapping workforce and a longer economical durability of the trees, compared to tapping at $\mathrm{d} 4$. The intensification of hormonal stimulation applied to lower tapping frequencies (d6) did not have any negative incidence on the clone PB 235. However, it has promoted tapping panel dryness in tapping at $\mathrm{d} 4$ frequency.
\end{abstract}


Keywords: Côte d'Ivoire, harvest, latex, tapping panel dryness, technology

\section{Résumé}

Le clone PB 235 d'Hevea brasiliensis est caractérisé par une croissance rapide, une homogénéité, une écorce propice à la saignée. Cependant, il est sensible aux technologies de récolte de latex très intenses. Cette étude vise à déterminer l'incidence de fortes fréquences de stimulation sur deux régimes de saignée descendante du clone PB 215: faible (d6) ou modéré (d4). Les hévéas ont été plantés à la densité de 510 arbres/ha $(7 \mathrm{~m} \mathrm{x}$ $2,8 \mathrm{~m}$ ), selon un dispositif en blocs aléatoires complets. Les fréquences de saignée d6, avec 8 et 12 stimulations hormonales, et d4, stimulée 0 et 8 fois par an, ont été évaluées. L'intensification de la stimulation hormonale en d6 n'a eu aucun effet bénéfique sur le rendement en caoutchouc sec des hévéas. En d4, la stimulation hormonale a entraîné une diminution de la teneur en saccharose du latex favorisant une augmentation significative du rendement en caoutchouc sec. Toutefois, elle a contribué à augmenter le taux d'encoche sèche des arbres. En saignée modérée, l'application de la stimulation hormonale a permis de distinguer les deux technologies de récolte du latex. Mais, en d6, les deux traitements ont été similaires indépendamment de la stimulation. La saignée en d6 favorise une réduction de la main d'œuvresaigneur et une plus longue durée de vie économique des arbres, comparée à la saignée en d4. L'intensification de la stimulation hormonale en d6 n'a eu aucune incidence négative sur le clone PB 235. Cependant, elle a favorisé ce phénomène en saignée modérée (d4).

Mots-clés : Côte d'Ivoire, encoche sèche, latex, récolte, technologie

\section{Introduction}

L'hévéa, de son nom botanique, Hevea brasiliensis Muell. Arg., est une plante pérenne cultivée pour son latex qui est une source de caoutchouc naturel (Rajagopal et al., 2003). La récolte du latex est faite suite à une incision dans l'écorce du tronc de l'arbre, en tenant compte de la maturité physiologique de l'arbre (Obouayeba, 2005 ; Soumahin, 2010). Cette maturité survient lorsque l'arbre a atteint $50 \mathrm{~cm}$ de circonférence, à une hauteur de $1 \mathrm{~m}$ au-dessus du sol, au moins 6 ans après sa plantation (Obouayeba, 2005). La production de latex ou caoutchouc peut être accrue par la saignée elle-même ou encore par la stimulation hormonale. En effet, ces deux techniques permettent de prolonger l'écoulement du latex après saignée et augmentent, par conséquent, le rendement des arbres (Dick et al., 2014). Cependant, les systèmes intensifs de récolte du latex dus à des fréquences élevées de saignée et/ou de stimulation hormonale sont causes de perturbation ou d'une absence totale d'écoulement de latex, suite à la saignée (Okoma et al., 2011). 
Le PB 235 est l'un des clones les plus étudiés en raison de sa croissance rapide, son homogénéité et son haut niveau de production de caoutchouc. Pourtant, il est sensible à l'encoche sèche. Les fréquences, de saignée et/ou de stimulation hormonale, modérées ou faibles peuvent être envisageables en vue d'une récolte plus pérenne du latex du clone PB 235, et surtout de faire face à certaines contraintes socio-économiques telles que la cherté de la main d'œuvre-saigneur, les prix non incitateurs, etc. (Soumahin et al., 2010). L'objectif de ce travail a été d'étudier deux régimes de saignée faible et modérée et quatre fréquences de stimulation hormonale pendant la saignée descendante du clone PB 215. Considérant les paramètres agronomiques et biochimiques les plus importants, cette étude visait à :

- comparer deux fréquences de saignée faible (d6) et modérée (d4) ;

- évaluer l'incidence de l'intensification de la stimulation pour chaque fréquence de saignée ;

- évaluer l'intérêt de ces fréquences de saignée en situations de contraintes socio-économiques.

\section{Matériel et méthodes \\ Milieu d'étude}

Le présent travail a été réalisé à Anguédédou, au sud-est de la Côte d'Ivoire, pays d'Afrique de l'ouest. Les coordonnées géographiques du pays sont $2^{\circ} 30$ et $8^{\circ} 30$ de longitude Ouest, $4^{\circ} 30$ et $10^{\circ} 30$ de latitude Nord. Il est caractérisé par deux grands types de paysages végétaux : l'un marqué par la forêt et l'autre par la savane (Monnier, 1983). Le domaine hévéicole s'étend de la basse Côte d'Ivoire à la zone forestière de l'ouest montagneux, puis à l'est. La végétation de la zone d'Anguédédou est composée de forêt dense ombrophile. Le régime pluviométrique est bimodal : d'avril à juin et de septembre à novembre, avec une moyenne annuelle se situant autour de 1600 $\mathrm{mm}$. La température moyenne mensuelle est de $25,5^{\circ} \mathrm{C}$ (Brou, 2005).

\section{Matériel végétal}

Le clone Prang Besar 235 (PB 235) d'Hevea brasiliensis est issu du croisement PB 5/51 x PB S/78 (Chapuset, 2001). Il a été créé en 1955 en Malaisie, plus précisément dans la localité de Prang Besar d'où il tire son nom. Il se caractérise par une homogénéité et un tronc assez droit et régulier. Son écorce vierge répond bien à la saignée. Sa croissance est rapide et demeure bonne en cours de saignée. Son architecture est favorable à la valorisation secondaire du bois en fin de vie économique (Chapuset, 2001).

\section{Dispositif expérimental et traitements}

Le dispositif expérimental est composé de blocs de Fisher complètement aléatoires avec 4 traitements (technologies de récolte du latex) 
et 4 répétitions. Le clone PB 235 a été planté à la densité de 510 arbres par hectare $(7 \mathrm{~m}$ x 2,80 m). Chaque parcelle élémentaire comportait 45 hévéas, soit 180 (45 x 4) arbres par traitement, correspondant à 720 individus étudiés. Le choix des arbres expérimentés a été fait en tenant compte de leur état sanitaire, de l'homogénéité, de leur circonférence, de la régularité du tronc et de la production à blanc (non stimulée). L'expérimentation a débuté avec l'ouverture des hévéas et elle s'est déroulée pendant dix années de saignée sur le panneau bas. Les traitements ou technologies de récolte du latex étudiées sont présentées dans le Tableau 1.

Tableau 1. Technologies de récolte du latex étudiées sur les hévéas du clone PB 235 au cours de dix années de saignée descendante en continu

\begin{tabular}{lc}
\hline \multicolumn{1}{c}{ Traitements } & $\begin{array}{c}\text { Nombre de } \\
\text { saignées par an }\end{array}$ \\
\hline $\mathrm{S} / 2 \mathrm{~d} 4$ non stimulé & 78 \\
$\mathrm{~S} / 2 \mathrm{~d} 46 \mathrm{~d} / 7 . \mathrm{ET} 2.5 \% \mathrm{~Pa} 1(1) 8 / \mathrm{y}(\mathrm{m})$ & 78 \\
$\mathrm{~S} / 2 \mathrm{~d} 66 \mathrm{~d} / 7 . \mathrm{ET} 2.5 \% \mathrm{~Pa} 1(1) 8 / \mathrm{y}(\mathrm{m})$ & 52 \\
$\mathrm{~S} / 2 \mathrm{~d} 66 \mathrm{~d} / 7 . \mathrm{ET} 2.5 \% \mathrm{~Pa} 1(1) 12 / \mathrm{y}(3 \mathrm{w})$ & 52 \\
\hline
\end{tabular}

Les traitements ont été décrits selon la notation internationale des technologies de récolte du latex de l'hévéa (Vijayakumar, 2008 ; Vijayakumar et al., 2009).

La signification du traitement $\mathrm{n}^{\circ} 3$, par exemple, est que la saignée est effectuée en demi-spirale descendante $(\mathrm{S} / 2)$ tous les 6 jours (d6), avec un jour de repos dans la semaine $(6 \mathrm{~d} / 7)$. La stimulation a été effectuée avec un mélange d'huile de palme et d'Ethrel, à la dose de 2,5\% d'Ethephon (ET2.5\%). Un gramme de ce mélange a été appliqué sur panneau (Pa), suivant une bande de $1 \mathrm{~cm}$ de large comprenant l'encoche de saignée (Pa1(1)), à raison de 8 applications par an (12/y), à intervalles d'un mois $(\mathrm{m})$. Le traitement $\mathrm{n}^{\circ}$ 4 est défini pareillement au $\mathrm{n}^{\circ} 3$, à la différence que la stimulation est effectuée 12 fois par an (12/y), à intervalles de 3 semaines (3w).

La saignée des hévéas étudiés a été effectuée tous les mois de chaque année par un seul saigneur, à l'aide d'un couteau de saignée. La matière active de l'Ethrel est l'acide chloro-2- éthylphosphonique ou Ethephon. L'application du produit stimulant a été réalisée à l'aide d'un pinceau.

\section{Mesures effectuées}

La production de caoutchouc a été enregistrée arbre par arbre, à raison d'une pesée chaque mois. Cette production représentant la masse de matière fraîche a été collectée au champ à l'état coagulé. Elle a ensuite été exprimée 
en grammes par arbre et par saignée $(\mathrm{g} / \mathrm{a} / \mathrm{s})$ et en kilogrammes par hectare par an $(\mathrm{kg} / \mathrm{ha} / \mathrm{an})$ de caoutchouc sec.

La circonférence du tronc de chaque arbre étudié a été mesurée, une fois par an, à une hauteur de 1,70 m du sol, lors de la mise en saignée, puis en fin de cycle physiologique, avant le début de la saison sèche. La croissance moyenne en épaisseur du tronc des hévéas a été exprimée en centimètres par an (cm/an).

La méthode du Micro Diagnostic Latex (MDL) mise au point par Jacob et al. (1988) a permis de déterminer, une fois par an, la valeur de l'extrait sec $(\mathrm{ExS})$ en pourcentage et de doser les teneurs en saccharose (Sacc), en phosphore inorganique (Pi) et en groupements thiols ( $\mathrm{R}-\mathrm{SH}$ ) du latex, exprimées en millimolaire $(\mathrm{mM})$. L'interprétation ou la signification des données des paramètres biochimiques est fondée sur les valeurs de référence présentées dans le (Tableau 2). En effet, le taux d'extrait sec renseigne sur la viscosité du latex. Il influence fortement l'écoulement du latex. Les teneurs en saccharose et en phosphore inorganique renseignent respectivement sur la disponibilité des réserves hydrocarbonées intralaticifères directement utilisables pour la biosynthèse du caoutchouc et sur l'activité métabolique énergétique des laticifères. Le niveau de leur teneur en groupements thiols traduit le degré de stabilité du latex.

Tableau 2. Valeurs de référence des quatre paramètres biochimiques les plus importants du latex (Jacob et al., 1987)

\begin{tabular}{lcccc}
\hline $\begin{array}{c}\text { Niveau } \\
\text { des teneurs }\end{array}$ & \multicolumn{4}{c}{ Paramètres biochimiques du latex } \\
& Ex) & $\begin{array}{c}\text { Sacc } \\
(\mathbf{m M})\end{array}$ & $\mathbf{P i}(\mathbf{m M})$ & $\begin{array}{c}\text { R-SH } \\
(\mathbf{m M})\end{array}$ \\
\hline Très élevé & $>43$ & $>12$ & $>25$ & $>0,90$ \\
Elevé & $38-43$ & $9-12$ & $20-25$ & $0,80-0,90$ \\
Moyen & $33-38$ & $6-9$ & $15-20$ & $0,6-0,8$ \\
Faible & $29-33$ & $4-6$ & $10-15$ & $0,5-0,6$ \\
Très faible & $\leq 29$ & $\leq 4$ & $\leq 10$ & $\leq 0,50$ \\
\hline
\end{tabular}

ExS : taux moyen d'extrait sec du latex ; \% : pourcentage ; mM : millimolaire ; Sacc (mM) : teneur moyenne en saccharose du latex; $\mathrm{Pi}(\mathrm{mM})$ : teneur moyenne en phosphore inorganique du latex; R-SH (mM) : teneur moyenne en groupements thiols du latex.

L'état sanitaire des hévéas a également été déterminé, avant le début de la saison sèche, à l'aide de la méthode d'estimation visuelle de l'encoche sèche de Van de Sype (1984). Les pourcentages de longueur totale d'encoche malade (LEM) et d'arbres totalement secs (ArbS), qui traduisent l'intensité de ce syndrome, ont été déterminés. 


\section{Analyse statistique}

Les valeurs des paramètres agronomiques (production de caoutchouc, de croissance en épaisseur du tronc) et biochimiques (profil physiologique) et de l'encoche sèche (LEM et ArbS) ont été modélisées par une analyse de variance (ANOVA), à l'aide du logiciel XLSTAT-Pro7.5.3. Le niveau de signification des différences entre les moyennes a été estimé par le test de Duncan au seuil de 5\%. Une analyse des composantes principales a été réalisée, à l'aide du logiciel Statistica, version 7, afin d'établir les rapports entre les paramètres étudiés et entre ceux-ci et les différents traitements. Ce logiciel a également permis d'effectuer une Classification Hiérarchique Ascendante (CHA) en vue de repartir les individus dans des classes homogènes.

\section{Résultats}

Incidence des technologies de récolte du latex sur la production de caoutchouc et la croissance en épaisseur du tronc des hévéas

L'analyse des données selon le test de Duncan, au seuil de 5\%, a révélé des différences entre les traitements au regard des valeurs de production de caoutchouc exprimées en g/a/s (Tableau 3). La faible fréquence de saignée (d6) a enregistré des productions $(69-73 \mathrm{~g} / \mathrm{a} / \mathrm{s})$ significativement supérieures à celles de la saignée modérée (51-57 g/a/s). Cependant, pour une même fréquence de saignée (faible ou modérée), il n’y a aucune différence entre les valeurs de production de caoutchouc et des technologies de récolte du latex, quelle que soit la fréquence de la stimulation hormonale.

Concernant la production de caoutchouc en $\mathrm{kg} / \mathrm{ha} / \mathrm{an}$, deux groupes de traitements ont été distingués. Le premier groupe est représenté par la saignée modérée stimulée 8 fois par an (d4 8/y) qui a enregistré la production de caoutchouc la plus élevée $(1783 \mathrm{~kg} / \mathrm{ha} / \mathrm{an})$. Le second groupe, composé des autres traitements (témoin $\mathrm{d} 40 / \mathrm{y}, \mathrm{d} 68 / \mathrm{y}$ et $\mathrm{d} 6 \mathrm{12} / \mathrm{y}$ ), a engendré des productions similaires variant de 1441 à $1591 \mathrm{~kg} / \mathrm{ha} / \mathrm{an}$ (Tableau 3).

Les différentes technologies de récolte du latex n'ont pas eu d'effet notoire sur les valeurs de circonférence du tronc des arbres qui sont toutes statistiquement identiques et comprises entre 59,8 et 61,7 cm (Tableau 3). 
Tableau 3. Valeurs moyennes de la production de caoutchouc et de la croissance en épaisseur du tronc des hévéas du clone PB 235 soumis à différents régimes de saignée et de stimulation au cours de dix années de saignée descendante en continu

\begin{tabular}{|c|c|c|c|}
\hline $\begin{array}{c}\text { Traitements } \\
\mathrm{S} / 26 \mathrm{~d} / 7_{0 .} \text { ET2.5\% }\end{array}$ & $\mathrm{g} / \mathrm{a} / \mathrm{s}$ & $\mathrm{Kg} / \mathrm{ha} / \mathbf{a n}$ & $\begin{array}{c}\text { Circ. à } 1,70 \mathrm{~m} \\
\text { du sol }(\mathrm{cm})\end{array}$ \\
\hline d4 non stimulé & $51 \mathrm{~b}$ & $1591 \mathrm{~b}$ & $61,7 \mathrm{a}$ \\
\hline $\mathrm{d} 4 \mathrm{~Pa} 1(1) 8 / y(m)$ & $57 \mathrm{~b}$ & $1783 \mathrm{a}$ & 59,8 a \\
\hline d6 Pa1(1) $8 / y(m)$ & $69 \mathrm{a}$ & $1441 \mathrm{~b}$ & $60,5 \mathrm{a}$ \\
\hline $\mathrm{d} 6 \mathrm{~Pa} 1(1) 12 / \mathrm{y}(3 \mathrm{w})$ & $73 \mathrm{a}$ & $1515 \mathrm{~b}$ & $60,2 \mathrm{a}$ \\
\hline
\end{tabular}

g/a/s : grammes par arbre et par saignée ; kg/ha/an : kilogrammes par hectare et par an ; Circ : Croissance en épaisseur. Dans une même colonne, les moyennes suivies de la même lettre ne sont pas significativement différentes (test de Duncan à 5\%).

Effets des technologies de récolte du latex sur le taux d'extrait sec, les teneurs en saccharose, en phosphore inorganique et en groupements thiols du latex des hévéas

L'analyse des résultats des paramètres biochimiques est basée ici sur les valeurs de référence présentées dans le Tableau 2. Le taux d'extrait sec, les teneurs en phosphore inorganique et en composés thiols, n'ont pas été influencés par les différents traitements (Tableau 4). Ces valeurs ont été équivalentes, quelles que soient la fréquence de saignée et l'intensité de la stimulation. Cependant, en s'appuyant sur les valeurs de référence (Tableau 2), la teneur en saccharose des arbres témoins non stimulés $(13 \mathrm{mM})$ a été très élevée. Les teneurs des autres traitements stimulés, comprises entre 7,8 et 8,6 $\mathrm{mM}$ ), ont été d'un niveau moyen (Tableaux 2 et 4). Quant aux taux d'extraits secs de l'ensemble des arbres étudiés, ils ont été très élevés (47,2 à 49,3\%).

Tableau 4.Valeurs moyennes du profil physiologique du clone PB 235 soumis à différents régimes de saignée et de stimulation au cours de dix années de saignée descendante en continu

\begin{tabular}{lllll}
\hline $\begin{array}{c}\text { Traitements } \\
\text { S/2 6d/7 ET2.5\% }\end{array}$ & ExS (\%) & $\begin{array}{r}\text { Sacc } \\
(\mathbf{m M})\end{array}$ & Pi (mM) & $\begin{array}{c}\text { R-SH } \\
(\mathbf{m M})\end{array}$ \\
\hline d4 non stimulé & $48,8 \mathrm{a}$ & $13,0 \mathrm{a}$ & $26,2 \mathrm{a}$ & $0,79 \mathrm{a}$ \\
d4 Pa1(1) $8 / \mathrm{y}(\mathrm{m})$ & $47,2 \mathrm{a}$ & $7,8 \mathrm{~b}$ & $27,1 \mathrm{a}$ & $0,66 \mathrm{a}$ \\
d6 $\mathrm{Pa} 1(1) 8 / \mathrm{y}(\mathrm{m})$ & $49,3 \mathrm{a}$ & $8,6 \mathrm{~b}$ & $23,6 \mathrm{a}$ & $0,63 \mathrm{a}$ \\
d6 $\mathrm{Pa} 1(1) 12 / \mathrm{y}(3 \mathrm{w})$ & $49,1 \mathrm{a}$ & $8,0 \mathrm{~b}$ & $24,8 \mathrm{a}$ & $0,56 \mathrm{a}$ \\
\hline
\end{tabular}

ExS : taux moyen d'extrait sec du latex ; \% : pourcentage ; $\mathrm{mM}$ : millimolaire ; Sacc (mM) : teneur moyenne en saccharose du latex; $\mathrm{Pi}(\mathrm{mM})$ : teneur moyenne en phosphore inorganique du latex; R-SH (mM) : teneur moyenne en groupements thiols du latex. Dans une même colonne, les moyennes suivies de la même lettre ne sont pas significativement différentes (test de Duncan à 5\%). 
Les teneurs en Pi du faible régime de saignée (d6) ont été de 23,6 mM et de $24,8 \mathrm{mM}$ respectivement pour 8 et 12 stimulations annuelles. Elles ont donc été d'un niveau élevé, tandis que celles de la fréquence d4 (26,2 et 27,1 $\mathrm{mM}$ ) ont été très élevées (Tableaux 2 et 4 ). La teneur en R-SH de la saignée en d6 stimulée 12 fois par an $(0,53 \mathrm{mM})$ est d'un niveau faible, alors que celles des autres traitements $(0,63-0,79 \mathrm{mM})$ ont été d'un niveau moyen.

\section{Influences des technologies de récolte du latex sur le taux d'encoche sèche des hévéas}

Le taux d'encoche sèche a été exprimé par le taux de longueur d'encoche malade (LEM) et le pourcentage d'arbres totalement secs (ArbS). Il existe des différences significatives entre les différents traitements, aussi bien au niveau du taux de LEM que du pourcentage des arbres totalement secs (Tableau 5).

Tableau 5. Taux moyens d'encoche sèche des hévéas du clone PB 235 soumis à différents régimes de saignée et de stimulation au cours de dix années de saignée descendante en continu

\begin{tabular}{lcc}
\hline \multicolumn{1}{c}{ Traitements } & \multicolumn{2}{c}{ Taux d'encoche sèche (\%) } \\
$\mathrm{S} / 26 \mathrm{~d} / \mathrm{m}_{\mathrm{a}}$ ET2.5\% & LEM & ArbS \\
\hline $\mathrm{d} 4$ non stimulé & $7,8 \mathrm{~b}$ & $2,1 \mathrm{ab}$ \\
$\mathrm{d} 4 \mathrm{~Pa} 1(1) 8 / \mathrm{y}(\mathrm{m})$ & $19,3 \mathrm{a}$ & $3,7 \mathrm{a}$ \\
$\mathrm{d} 6 \mathrm{~Pa} 1(1) 8 / \mathrm{y}(\mathrm{m})$ & $9,5 \mathrm{~b}$ & $1,7 \mathrm{~b}$ \\
$\mathrm{~d} 6 \mathrm{~Pa} 1(1) 12 / \mathrm{y}(3 \mathrm{w})$ & $9,3 \mathrm{~b}$ & $0,9 \mathrm{~b}$ \\
\hline
\end{tabular}

LEM : Longueur d'encoche malade ; ArbS : Arbres totalement secs. Dans une même colonne, les moyennes suivies de la même lettre ne sont pas significativement différentes (test de Duncan à 5\%).

La saignée modérée stimulée 8 fois par an (d4 8/y) a induit le taux le plus élevé $(19,3 \%)$ de LEM, par rapport aux autres traitements (y compris le témoin non stimulé) qui ont des valeurs similaires $(7,8-9,5 \%)$. Au niveau du pourcentage d'arbres totalement secs, la valeur enregistrée avec la saignée en d4 à 8 stimulations par an $(3,7 \%)$ a été la plus importante. Quant aux autres technologies de récolte du latex, elles ont induit des taux d'arbres secs statistiquement identiques et compris entre $0,9-2,1 \%$.

Incidence de la fréquence de saignée sur la main d'œuvre (saigneurs) et la durée de vie économique du panneau bas des hévéas

Ces résultats ont concerné le nombre annuel de coups de saignée, le niveau de réduction du besoin en main d'œuvre-saigneur et la durée de vie économique des arbres (Tableau 6). La saignée modérée (d4) a nécessité 78 
saignées annuelles contre 52 pour la faible fréquence de saignée (d6). La d6 a permis une réduction de 33,33\% du besoin en main d'œuvre-saigneur, par rapport à la saignée modérée (d4). Quant à la durée de vie économique des arbres sur le panneau bas, elle a été de 26 ans, avec la saignée tous les quatre jours (d4) et de 31 ans, pour la saignée tous les six (d6) jours.

Tableau 6. Taux de réduction de la main d'œuvre-saigneur et durée de vie économique du panneau bas des hévéas selon la fréquence de saignée

\begin{tabular}{llcc}
\hline $\begin{array}{c}\text { Traitements } \\
\mathrm{S} / 26 \mathrm{~d} / 7 \mathrm{~m} \text { ET2.5\% }\end{array}$ & NS & $\begin{array}{c}\text { RMOS } \\
\text { (\%) }\end{array}$ & $\begin{array}{c}\text { DVEPB } \\
\text { (ans) }\end{array}$ \\
\hline $\mathrm{d} 4$ non stimulé & 78 & 0 & 26 \\
$\mathrm{~d} 4 \mathrm{~Pa} 1(1) 8 / \mathrm{y}(\mathrm{m})$ & 78 & 0 & 26 \\
$\mathrm{~d} 6 \mathrm{~Pa} 1(1) 8 / \mathrm{y}(\mathrm{m})$ & 52 & 33,33 & 31 \\
$\mathrm{~d} 6 \mathrm{~Pa} 1(1) 12 / \mathrm{y}(3 \mathrm{w})$ & 52 & 33,33 & 31 \\
\hline
\end{tabular}

NS : nombre de saignées par an ; RMOS ; taux de réduction de la main d'œuvre-saigneur ; DVEPB : durée de vie économique sur le panneau bas.

\section{Valeurs propres et pourcentage de variation des différents axes de l'analyse des composantes principales}

Les valeurs propres et le pourcentage de variance de chaque axe (composante) sont présentés dans le Tableau 7. En tenant compte du critère de Kaiser, tout axe dont la valeur propre est supérieure à 1 doit être retenu pour l'analyse. Par conséquent, seuls les deux premiers axes (axes 1 et 2) ont été retenus pour définir la variabilité totale des traitements. Ces deux axes permettent d'expliquer $97,55 \%$ de la variabilité totale. Le premier axe restitue 65,14 de cette variabilité, tandis que le second n'en exprime que $32,41 \%$. Le troisième axe ne peut être retenu puisque sa valeur propre est de 0,293 et donc inférieure à 1 .

Tableau 7. Valeurs propres et pourcentage de variation des trois premiers axes de l'analyse des composantes principales

\begin{tabular}{lccc}
\hline Composantes & Axe 1 & Axe 2 & Axe 3 \\
Variance propre & 7,817621 & 3,889224 & 0,293155 \\
\% variance totale & 65,14684 & 32,41020 & 2,44296 \\
\% variance totale & 65,1468 & 97,5570 & 100 \\
\hline
\end{tabular}

\section{Corrélations entre les paramètres étudiés et les axes 1 et 2}

Deux grands groupes de paramètres peuvent être distingués relativement à la formation de chacun des deux axes retenus (Tableau 8) :

- le groupe 1 est composé des variables grammes par arbre et par saignée $(\mathrm{g} / \mathrm{a} / \mathrm{s})$, phosphore inorganique $(\mathrm{Pi})$, kilogrammes par hectare et par an (kg/ha/an), extrait sec (ExS), arbres totalement secs (ArbS), nombre de saignées (NS), réduction de la main d'œuvre-saigneur (RMOS) et durée de vie économique des arbres sur le panneau bas (DVEPB) qui ont contribué à la 
formation de l'axe 1 (Tableau 8). Toutefois, les paramètres g/a/s, ExS, RMOS, DVEPB sont corrélés négativement à cet axe, alors que les autres, de ce même groupe lui sont corrélés positivement (Tableau 8, Figure 1) ;

- le second groupe réunit les variables circonférence (Circ), teneurs en saccharose (Sacc), en groupements thiols ( $\mathrm{R}-\mathrm{SH})$ et longueur d'encoche malade (LEM) qui caractérisent l'axe 2. Cependant, seule la LEM est corrélée positivement à cet axe (Tableau 8 , Figure 1).

Tableau 8 : Corrélations entre les paramètres étudiés et les axes 1 et 2

\begin{tabular}{lcc}
\hline \multicolumn{1}{c}{ Variables } & \multicolumn{2}{c}{ Composantes } \\
& Axe l & Axe 2 \\
\hline $\mathrm{g} / \mathrm{a} / \mathrm{s}$ & $-0,9092^{*}$ & 0,4069 \\
$\mathrm{Kg} / \mathrm{ha} / \mathrm{an}$ & $0,8928^{*}$ & 0,4227 \\
$\mathrm{Circ}$ & 0,1288 & $-0,9917^{*}$ \\
$\mathrm{ExS}$ & $-0,8238^{*}$ & $-0,5667$ \\
$\mathrm{Sacc}$ & 0,3501 & $-0,9357^{*}$ \\
$\mathrm{Pi}$ & $0,9399^{*}$ & 0,1364 \\
$\mathrm{Rsh}$ & 0,6851 & $-0,7043^{*}$ \\
$\mathrm{LEM}$ & 0,5913 & $0,7963^{*}$ \\
$\mathrm{ArbS}$ & $0,8666^{*}$ & 0,3778 \\
$\mathrm{NS}$ & $0,9870^{*}$ & $-0,1564$ \\
$\mathrm{RMOS}$ & $-0,9870^{*}$ & 0,1564 \\
$\mathrm{DVEPB}$ & $-0,9870^{*}$ & 0,1564 \\
\hline \multicolumn{3}{c}{}
\end{tabular}

g/a/s : grammes par arbre et par saignée $; \mathrm{Kg} / \mathrm{ha} / \mathrm{an}$ : Kilogrammes par hectare et par an ;

ExS : extrait sec ; Sacc : saccharose ; Pi : phosphore inorganique ; R-SH : groupements thiols ; LEM : Longueur d'encoche malade ; ArbS : arbres totalement secs ; NS : nombre de saignées ; RMOS : réduction de la main d'œuvre-saigneur ; DVEPB : durée de vie économique du panneau bas.

\section{Répartition des technologies de récolte du latex (individus) dans le plan formé par les axes 1 et 2}

Le traitement témoin (d4 0/y) se situe dans le quart du plan correspondant à la partie négative de l'axe 2 et positive de l'axe 1 (Figure 2). Les technologies d6 8/y et d6 12/y appartiennent respectivement à la partie positive et à la partie négative de l'axe 2. Elles sont toutes deux dans la partie négative de l'axe 1. Cependant, ces individus sont les plus proches sur le plan, avec une distance euclidienne de 1,64 (Figure 2, Tableau 9). Le traitement d4 8/y est situé dans le quart du plan correspondant à la fois aux parties positives des axes 1 et 2 (Tableau 8, Figure 1). Sur le plan, les individus les plus éloignés ont été les traitements d4 8/y et d6 8/y, avec une distance euclidienne de 5,74 (Tableau 9). 
Tableau 9. Matrice de proximité des technologies de récolte du latex (distance euclidienne)

\begin{tabular}{lcccc}
\hline $\begin{array}{l}\text { Traitements } \\
\text { ou individus }\end{array}$ & $\begin{array}{c}\text { d4 non } \\
\text { stimulé }\end{array}$ & d4 $\mathbf{8} / \mathbf{y}$ & $\mathbf{d 6} \mathbf{8} / \mathbf{y}$ & $\mathbf{d 6} \mathbf{1 2 / \mathbf { y }}$ \\
\hline d4 non stimulé & 0 & & & \\
d4 $8 / y$ & 4,846 & 0 & & \\
d6 $8 / y$ & 4,958 & 5,748 & 0 & \\
d6 $12 / y$ & 5,397 & 5,614 & 1,497 & 0 \\
\hline
\end{tabular}

d4 non stimulé : S/2 d4 non stimulé ; d4 8/y : S/2 d4 6d/7. ET2.5\%Pa1(1) 8/y(m) d6 8/y : S/2 d6 6d/7. ET2.5\%Pa1(1) 8/y(m) ; d6 12/y : S/2 d6 6d/7. ET2.5\%Pa1(1) 12/y(3w)

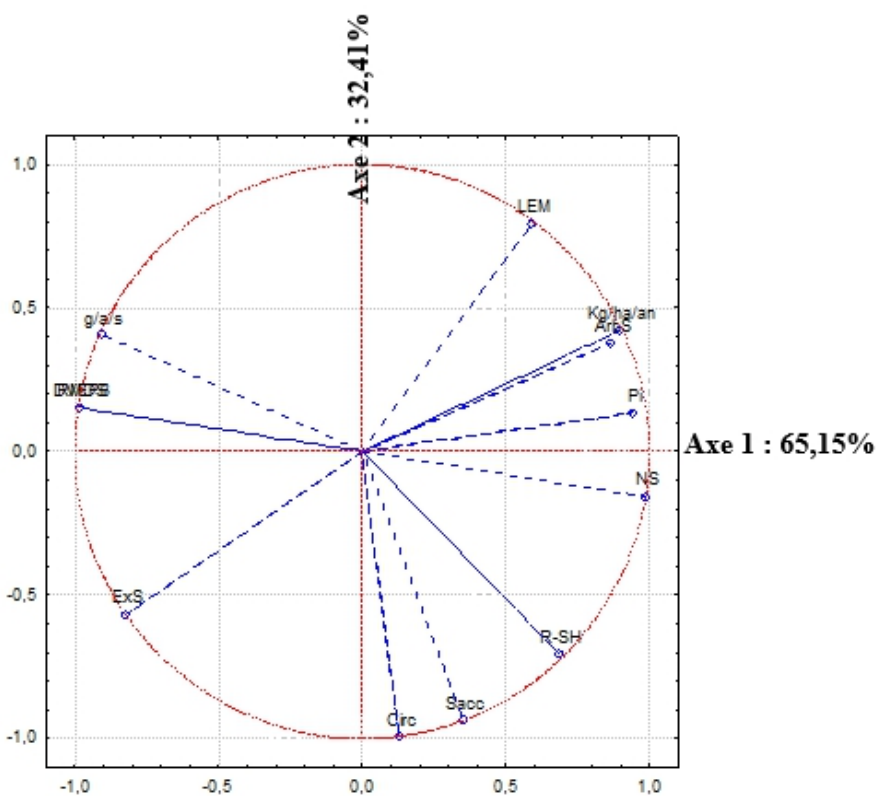

Figure 1. Répartition de l'ensemble des paramètres agronomiques et biochimiques dans le plan formé par les axes 1 et 2

$\mathrm{g} / \mathrm{a} / \mathrm{s}$ : grammes par arbre et par saignée ; $\mathrm{kg} / \mathrm{ha} / \mathrm{an}:$ kilogrammes par hectare et par an ; ExS : extrait sec ; Sacc : saccharose ; Pi : phosphore inorganique ; R-SH : groupements thiols ;

LEM : Longueur d'encoche malade ; ArbS : arbres totalement secs ; NS : nombre de saignées ; RMOS : réduction de la main d'œuvre-saigneur ; DVEPB : durée de vie économique sur le panneau bas.

\section{Corrélations entre l'ensemble des paramètres agronomiques et biochimiques étudiés}

Les corrélations (Tableau 10) qui existent entre les variables étudiées ont été négatives entre la production en $\mathrm{g} / \mathrm{a} / \mathrm{s}$ et le nombre annuel de saignée (NS), la production de caoutchouc en $\mathrm{kg} / \mathrm{ha} / \mathrm{an}$ et le taux d'extrait sec; le NS et la réduction de la main d'œuvre-saigneur (RMO), de même que la durée de vie économique des arbres sur le panneau bas (DVEPB). Par contre, des 
corrélations positives ont été révélées entre la production en $\mathrm{g} / \mathrm{a} / \mathrm{s}$ et la réduction de la main d'œuvre-saigneur (RMOS) ou la durée de vie économique des arbres sur le panneau bas (DVEPB), entre la circonférence du tronc (Circ) et la teneur en saccharose (Sacc) aussi bien qu'entre la réduction de la main d'œuvre-saigneur (RMOS) ou la durée de vie économique des arbres sur le panneau bas (DVEPB).

Tableau 10. Corrélations entre l'ensemble des paramètres agronomiques et biochimiques étudiés

\begin{tabular}{crrrrrrrrrrr}
\hline Variables & g/a/s & Kg/ha/an & Circ & ExS & Sacc & Pi & R-SH & LEM & ArbS & NS & RMOS DVEPB \\
\hline g/a/s & $\mathbf{1}$ & & & & & & & & & & \\
Kg/ha/an & $-0,626$ & $\mathbf{1}$ & & & & & & & & & \\
Circ & $-0,521$ & $-0,304$ & $\mathbf{1}$ & & & & & & & & \\
ExS & 0,517 & $\mathbf{- 0 , 9 7 7}$ & 0,456 & $\mathbf{1}$ & & & & & & & \\
Sacc & $-0,695$ & $-0,076$ & $\mathbf{0 , 9 7 3}$ & 0,241 & $\mathbf{1}$ & & & & & & \\
Pi & $-0,771$ & 0,946 & $-0,015$ & $-0,855$ & 0,215 & $\mathbf{1}$ & & & & & \\
Rsh & $-0,926$ & 0,285 & 0,787 & $-0,163$ & 0,891 & 0,490 & $\mathbf{1}$ & & & & \\
LEM & $-0,225$ & 0,845 & $-0,713$ & $-0,937$ & $-0,544$ & 0,624 & $-0,132$ & $\mathbf{1}$ & & & \\
ArbS & $-0,663$ & 0,883 & $-0,263$ & $-0,924$ & $-0,065$ & 0,764 & 0,388 & 0,855 & $\mathbf{1}$ & & \\
NS & $\mathbf{- 0 , 9 5 8}$ & 0,821 & 0,282 & $-0,725$ & 0,493 & 0,918 & 0,780 & 0,455 & 0,784 & $\mathbf{1}$ & \\
RMO & $\mathbf{0 , 9 5 8}$ & $-0,821$ & $-0,282$ & 0,725 & $-0,493$ & $-0,918$ & $-0,780$ & $-0,455$ & $-0,784$ & $\mathbf{- 1 , 0 0 0}$ & $\mathbf{1}$ \\
DVE & $\mathbf{0 , 9 5 8}$ & $-0,821$ & $-0,282$ & 0,725 & $-0,493$ & $-0,918$ & $-0,780$ & $-0,455$ & $-0,784$ & $\mathbf{- 1 , 0 0 0}$ & $\mathbf{1 , 0 0 0}$ \\
\hline
\end{tabular}

Les valeurs en gras sont différentes de 0 à un niveau de signification alpha $=0,05 ; \mathrm{g} / \mathrm{a} / \mathrm{s}$ : grammes par arbre et par saignée ; Kg/ha/an : Kilogrammes par hectare et par an ; ExS : extrait sec ; Sacc : saccharose ; Pi : phosphore inorganique ; R-SH : groupements thiols ;

LEM : Longueur d'encoche malade ; ArbS : arbres totalement secs ; NS : nombre de saignées ; RMOS : réduction de la main d'œuvre-saigneur ; DVEPB : durée de vie économique sur le panneau bas.

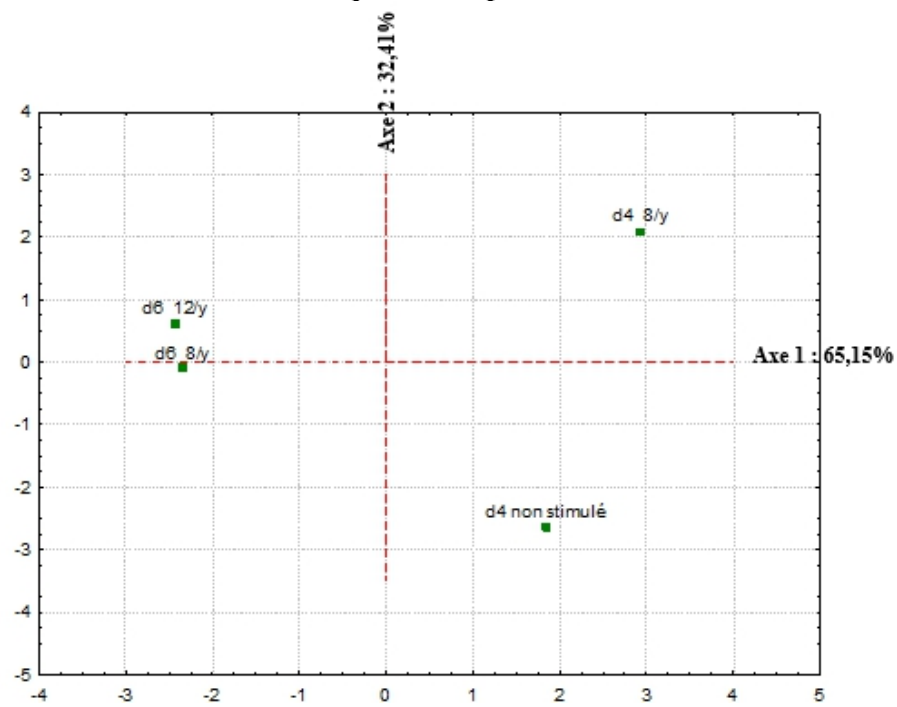

Figure 2. Projection des technologies de récolte dans le plan formé par les axes 1 et 2 
d4 non stimulé : S/2 d4 non stimulé ; d4 8/y : S/2 d4 6d/7. ET2.5\%Pa1(1) 8/y(m) d6 8/y : S/2 d6 6d/7. ET2.5\%Pa1(1) 8/y(m) ; d6 12/y : S/2 d6 6d/7. ET2.5\%Pa1(1) 12/y(3w)

$\mathrm{Au}$ vu des résultats des ACP (Figures 1 et 2), des corrélations entre variables (Tableau 10) et de la classification hiérarchique ascendante (Figure 3), il ressort que les technologies de récolte du latex peuvent être rassemblées en trois groupes homogènes. La séparation de ces groupes est assez nette à la troncature de 1,5 unité sur le dendrogramme (Figure 3). Ces groupes d'individus comportent chacun des variables caractéristiques. Le premier est constitué de la saignée en d6 (avec 8 et 12 stimulations) et des variables production en g/a/s, RMOS, DVEPB et ExS. Le second est représenté par la saignée non stimulée (d4 0/y) et caractérisé par les paramètres de circonférence du tronc, de teneurs en saccharose et en groupements thiols. Le troisième groupe est constitué par la saignée en $\mathrm{d} 4$ stimulée 8 fois qui réunit les variables nombre annuel de saignées, teneur en phosphore inorganique, production en $\mathrm{kg} / \mathrm{ha} / \mathrm{an}$, taux de LEM et d'arbres totalement secs.

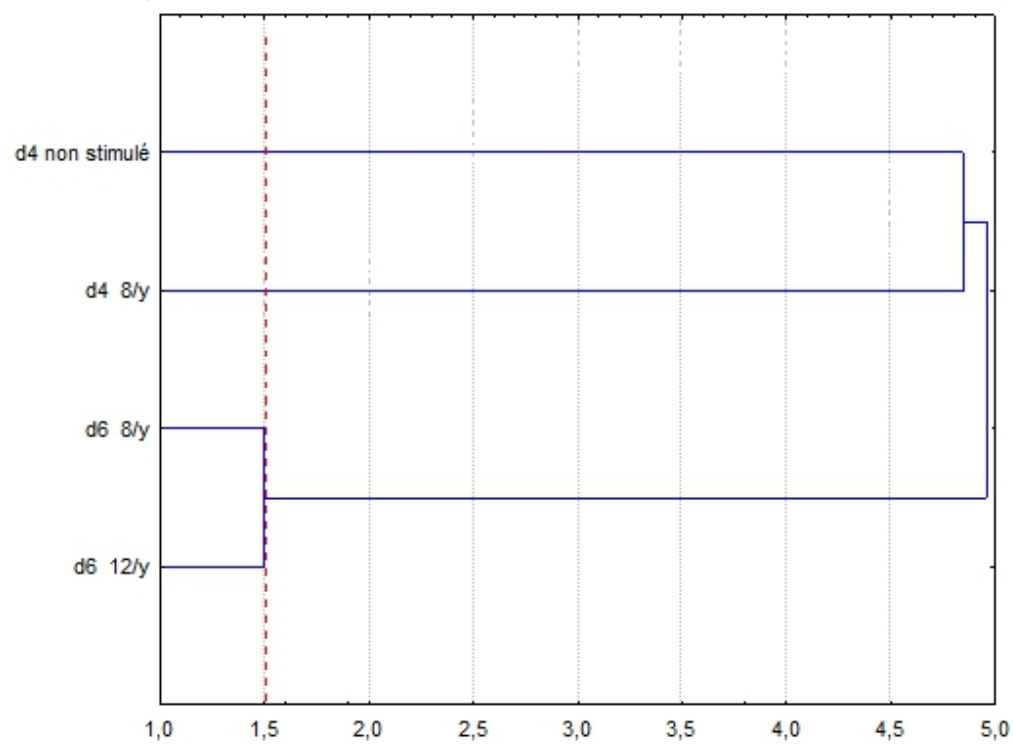

Figure 3. Regroupement des technologies de récolte du latex en classes homogènes, selon la méthode d'agrégation de Ward

\section{Discussion}

La faible fréquence de saignée (d6) a entrainé des productions de caoutchouc en $\mathrm{g} / \mathrm{a} / \mathrm{s}$ supérieures à celles de la fréquence modérée (d4). Toutefois, c'est plutôt la situation inverse qui est observée dans le cas de la production de caoutchouc exprimée en kg/ha/an. Autrement dit, moins les saignées sont fréquentes, plus la production de caoutchouc en g/a/s est importante. En effet, plus le délai entre deux saignées consécutives est important, plus il permet de compenser le latex récolté par une régénération du contenu des laticifères. 
La production de caoutchouc exprimée en $\mathrm{kg} / \mathrm{ha} / \mathrm{an}$, qui est liée au nombre de saignées par an, explique les rendements plus importants obtenus avec les technologies à régimes de saignée modérée (d4) par rapport à celles à régime de saignée faible (d6). Cette situation permet de conclure que le nombre de saignées par an est le paramètre le plus déterminant dans l'expression du rendement en caoutchouc des arbres. Le faible régime de saignée, qui est ici la d6, a un nombre annuel de saignées moins élevé que celui de la saignée modérée (d4). Ces résultats de la production de caoutchouc exprimée aussi bien en $\mathrm{g} / \mathrm{a} / \mathrm{s}$ qu'en $\mathrm{kg} / \mathrm{ha} / \mathrm{an}$ sont tous en accord avec les travaux de Diarrassouba et al. (2012) sur les clones PB 217 et PR 107 et ceux de Coulibaly et al. (2011 et 2017) sur le clone PB 235. Ces auteurs ont tous établi les mêmes rapports entre la fréquence de saignée et la production de caoutchouc des hévéas.

Concernant la croissance en épaisseur du tronc des arbres, l'absence de différences entre les technologies de récolte du latex traduit une très faible influence de la production de caoutchouc sur ce facteur. Des résultats similaires ont été déjà rapportés par Traoré et al. (2013), qui ont montré que chez le clone PB 217 l'antagonisme existant entre croissance et production de latex est d'une faible ampleur. Cependant, les travaux d'Obouayeba (2005) ont montré qu'il existe un phénomène de compétition et de répartition des assimilats entre l'élaboration de la biomasse primaire et la synthèse de caoutchouc. Selon cet auteur, plus la production de latex est importante, plus la croissance de l'hévéa est faible. Le niveau de l'antagonisme entre ces deux paramètres chez l'hévéa serait donc fonction de la technologie de récolte du latex pratiquée.

Les taux d'extrait sec du latex très élevés chez l'ensemble des hévéas résultent d'une intense activité biosynthétique des laticifères. Cette activité serait à l'origine d'une régénération efficace du latex, comme déjà rapporté par Soumahin et al. (2009) sur le clone PB 217, par Diarrassouba et al. (2012) sur le clone PR 107 et par Coulibaly et al. (2017) sur le clone PB 235.

Les teneurs en saccharose du latex, très élevées chez les hévéas non stimulés, indiquent un approvisionnement suffisant des laticifères en glucides, mais surtout couplé d'un métabolisme très peu activé. Le faible niveau de l'activité métabolique des laticifères dû à l'absence de stimulation hormonale explique le rendement en caoutchouc moins important que celui des arbres saignés à la même fréquence et stimulés 8 fois par an (d4 8/y). Les teneurs en saccharose du latex qui ont été d'un niveau moyen, chez l'ensemble des hévéas stimulés traduisent un métabolisme laticifère plus activé dû à l'effet de la stimulation hormonale. L'effet activateur de la saignée et de la stimulation sur la production de latex des hévéas a été déjà mis en évidence par Traoré $e t$ al. (2013). Les teneurs en Pi élevés et très élevées respectivement avec les fréquences de saignée d6 et d4 sont en lien avec la disponibilité de l'énergie 
nécessaire à l'activation du métabolisme laticigène. Cette énergie est plus importante chez les hévéas saignés en d4 que chez ceux saignés en d6.

Les teneurs en groupements thiols du latex des hévéas ont été d'un niveau moyen, à l'exception de la saignée d6 stimulée 8 fois par an. Ces teneurs traduisent une protection biologique des lutoïdes et une stabilité du latex des hévéas plus acceptables chez l'ensemble des arbres. Toutefois, le phénomène serait relativement moins important chez les hévéas saignés en d6 et stimulés 12 fois. Nos résultats sont conformes à ceux de Dick et al. (2014) à la fois sur les clones PB 235 et PB 260.

Les taux d'encoche sèche (LEM et arbres secs) plus importants enregistrés au niveau de la saignée en d4 stimulée, comparativement aux arbres non stimulés, indique que le phénomène est ici lié à la stimulation hormonale. Des résultats similaires ont déjà été rapportés par Obouayeba et al. (2010).

La faible fréquence de saignée (d6) a favorisé une réduction de la main d'œuvre- saigneur et une plus longue durée de vie économique des arbres par rapport aux saignées en $\mathrm{d} 4$. Ces résultats sont en accord avec les travaux de nombreux auteurs (Soumahin, 2010 ;Coulibaly et al., 2017) qui ont rapporté que pour une même longueur d'encoche de saignée, plus la fréquence de saignée est faible, plus la durée de vie économique des arbres est importante.

\section{Conclusion}

L'étude de l'effet des fortes fréquences de stimulation hormonale sur deux régimes (faible ou modéré) de saignée descendante du clone PB 215, a été menée durant dix années à Anguédédou, au sud-est de la Côte d'Ivoire. Elle a révélé que la fréquence de saignée modérée $(\mathrm{d} 4)$, par rapport à la d6, a induit une augmentation de la teneur en phosphore inorganique (Pi). Les faibles fréquences de saignée (d6) favorisent une réduction de la main d'œuvre-saigneur et une plus longue durée de vie économique des arbres par rapport aux saignées en $\mathrm{d} 4$.

L'intensification de la stimulation hormonale (passant de 8 à 12 fois par an), au niveau de la faible fréquence de saignée (d6), n'a aucun effet bénéfique sur le rendement en caoutchouc sec des hévéas. Elle n'a influencé ni la teneur en Saccharose du latex des hévéas ni la survenue de l'encoche sèche chez les hévéas. Pourtant, elle a induit une diminution relative des teneurs en groupements thiols (R-SH).

Au niveau des arbres saignés, suivant un régime modéré, trois fois par quinzaine (d4), l'apport de la stimulation (passant de 0 à 8 fois par an) a permis une augmentation significative du rendement en caoutchouc sec. La stimulation a entraîné une diminution de la teneur en saccharose (Sacc) du latex qui a favorisé la productivité des hévéas. Toutefois, elle a contribué à augmenter le taux d'encoche malade (LEM) des arbres. 
En ce qui concerne les conditions expérimentales, la stimulation hormonale n'a eu aucun effet, ni sur la croissance isodiamétrique du tronc, ni sur les taux d'extraits secs du latex des hévéas, ou encore sur la teneur en Pi du latex des hévéas.

L'apport de la stimulation hormonale, au niveau de la fréquence de saignée modérée $(\mathrm{d} 4)$, permet de distinguer les deux technologies de récolte $\mathrm{du}$ latex, avec respectivement 0 et 8 stimulations annuelles. Cependant, les deux traitements, avec saignées une fois par semaine (d6), sont similaires indépendamment de l'intensification de la stimulation ( 8 ou 12 fois par an).

L'intensification de la stimulation hormonale n'a aucune incidence négative sur la survenue de l'encoche sèche en faible régime de saignée (d6). Cependant, elle favorise ce phénomène en saignée modérée (d4). Les technologies de récolte du latex d4 8/y et d6 8/y se sont avérées les mieux adaptées. Toutefois, la d6 8/y permet de mieux faire face à une situation de manque de main d'œuvre-saigneur.

\section{References:}

1. Brou, Y. T. (2005). Climat, mutations socio-économiques et paysages en Côte d'Ivoire. Mémoire de synthèse des activités scientifiques présentée en vue de l'obtention de l'Habilitation à diriger des recherches. Université des Sciences et Technologies de Lille, $213 p$.

2. Chapuset, T. (2001). Description des clones étudiés à grande échelle, rapport CNRA-HEVEA n01/01-A, 22-23.

3. Coulibaly, L. F., Diarrassouba, M., Obouayeba, S., Yapi, G. C. V. \& Aké, S. (2011). Système d'exploitation en saignée inversée du clone PB 235 d'Hevea brasiliensis en Côte d'Ivoire. Journal of Animal \& Plant Sciences, 9(2): 1147-1160.

4. Coulibaly, L. F., Traoré, M. S., Soumahin, E. F., Obouayeba, S. \& Kéli, Z. J. (2017). Incidence des fréquences de saignées d3, d4 et d6, à faibles régimes de stimulation hormonale, sur le panneau descendant du clone PB 235 d'hévéa en Côte d'Ivoire. Int. J. Biol. Chem. Sci. 11(4): 1655-1668, 2017.

5. Dick, E. A., Traoré, M. S., Elabo, A. E. A., Soumahin, E. F., Assi, E. G. M., Atsin, O. G. J., Alle, Y. J., N'guessan, A. E. B., Kouamé, C. \& Obouayeba, S. 2014. Effets de différentes fréquences annuelles de stimulation éthylénique sur les paramètres agrophysiologiques et de sensibilité à l'encoche sèche d'Hevea brasiliensis au sud est de la Côte d'Ivoire : cas des clones PB 235 et PB 260 de la classe d'activité rapide. Int. J. Biol. Chem. Sci., 8(3): 956-974.

6. Diarrassouba, M., Soumahin, E. F., Coulibaly, L. F., N'guessan, A. E. B., Koulaka, E. D., Kouamé, C., Obouayeba, S. \& Aké, S. (2012). Latex harvesting technologies adapted to clones PB 217 and PR107 of 
Hevea brasiliensis Muell. Arg. of the slow metabolism class and of the socio-economic context in Côte d'Ivoire. Int. J. Biosci., 2(12): 125138.

7. Jacob, J. L., Serres, E., Prévot, J. C., Lacrotte, R., Vidal, A., Escbach, J. M. \& D'auzac, J. (1988). Mise au point du diagnostic latex chez l'hévéa. Agritrop, 12(12): 97-118.

8. Jacob, J. L., Lacrotte, R., Serres, E. \& Roussel, D. (1987). Les paramètres physiologiques du latex d'Hevea brasiliensis. Le diagnostic latex, ses bases, sa mise au point. IRCA, 41p.

9. Monnier, Y. (1983). Végétation. In : les atlas jeune Afrique Cote d'Ivoire. J. A, pp. 16-18.

10. Obouayeba, S. (2005). Contribution à la détermination de la maturité physiologique de l'écorce pour la mise en saignée d'Hevea brasiliensis Muell. Arg. (Euphorbiaceae): Normes d'ouverture. Thèse de Doctorat, Université de Côte d'Ivoire, Abidjan, 225p.

11. Obouayeba, S., Soumahin, E. F. \& Coulibaly, L. F. (2010). Low intensity tapping systems applied to clone PR 107 of Hevea brasiliensis (Muell. Arg.) in South-eastern Côte d'Ivoire: influence of the nature of the exploited bark and the position of tapping panel. Agric. Biol. J. N. Am., 1(5): 1106-1118.

12. Okoma, K. M., Dian, K., Obouayeba, S., Elabo, A. A. E. \& N'guetta, A. S. P. (2011). Seasonal variation of tapping panel dryness expression in rubber tree Hevea brasiliensis Muell. Arg. in Côte d'Ivoire. Agric. Biol. J. N. Am., 2(3): 559-569.

13. Rajagopal, R., Vijayakumar, K. R., Thomas, K. U. \& Karunaichamy, K. (2003). Yield response of Hevea brasiliensis (clone PB 217) to low frequency tapping. Proceeding of the international Workshop on exploitation technology, India, 127-139.

14. Soumahin, E. F. (2010). Optimisation des systèmes d'exploitation en hévéaculture par la réduction des intensités de saignée. Thèse de Doctorat de 1'Université de Cocody, 206p.

15. Soumahin, E. F, Obouayeba, S. \& Anno, A. P. (2009). Low tapping frequency with hormonal stimulation on Hevea brasiliensis clone PB 217 reduces tapping manpower requirement. Journal of Animal \& Plant Sciences, 2(3): 109-117.

16. Traoré, M. S., Dick, A. E., Elabo, A. E. A., Soumahin, E. F., Coulibaly, L. F., Camara, B., Allé, Y. J., N’guessan, A. E. B., Kouamé, C. \& Obouayeba, S. (2013). Effets de différentes fréquences annuelles de stimulation éthylénique sur les paramètres agrophysiologiques et la sensibilité à l'encoche sèche des clones d'Hevea brasiliensis au sudest de la Côte d'Ivoire: cas de la classe d'activité lente (PB 217). European Journal of Scientific Research, 114(4): 447-465. 
17. Van de Sype, H. (1984). The dry cut syndroms of Hevea brasiliensis, evolution, agronomical and physiological aspects. C.R. Coll. Expl. Physiol. Amél. Hévéa, ed., IRCA, CIRAD, Montpellier, France, pp. 227-249.

18. Vijayakumar, K. R. (2008). International revised notation for latex production technology. IRRDB Workshop of latex Harvesting Technologies, Sungai Buloh, Selangor, 5-8 May 2008, 20p.

19. Vijayakumar, K. R., Gohet, E., Thomas, K. U., Xiaodi, W., Sumarmadji, R. L., Thanh, DO K., Sopchoke, P., Karunaichamy, K. \& Said, M. (2009). International revised notation for latex harvest technology. International Rubber Research and Development Board (IRRDB), 19p. 